\title{
A healthier future requires policy informed by lifespan science
}

\author{
Shale L. Wong' ${ }^{1}$ and Vivek Balasubramaniam²
}

$\mathbf{L}$ ifespan developmental theory provides a framework and philosophy for understanding human aging. Growing evidence illustrates that even the earliest childhood development has effects on adult outcomes, beginning with processes such as cellular development in brain architecture $(1,2)$ and cardiac endothelial cell production (3) and resulting in lifelong health or chronic disease. Life course theory includes the familial, social, and environmental experiences that further shape development into adulthood, including adverse childhood experiences. $(4,5)$. The potent combination of discoveries in biological and social sciences demonstrates that the pathophysiology of adult disease begins in childhood and has lifelong implications. In this issue of Pediatric Research, Hammoud et al.(6) discuss the impact of maternal diabetes mellitus on infant growth and BMI through adolescence, and Kuwahara et al.(7) examine the association between childhood BMI and adult serum uric acid levels as a marker for chronic disease. This research illustrates the interconnectedness of disease and conditions across generations and the lifespan, and highlights a unique opportunity to translate lifespan science into intergenerational health policies that support and strengthen health of children, families, and communities and reduce the economic burden of chronic disease.

In Hammoud's work, we learn that prenatal maternal diabetes mellitus, type 2 , increases the risk of overweight and obesity in childhood that persists through $14 \mathrm{y}$ of growth. Recognizing the challenges of overcoming childhood obesity, the authors suggest that intergenerational approaches addressing maternal diabetes risk factors may decrease risk for excessive weight gain in their children. Policies that aim to decrease childhood obesity and disease most frequently target the child or their environment. Pediatricians discuss the child in the context of family, but rarely consider interventions to influence maternal self-behavior. The National Academy of Medicine (previously, Institute of Medicine) has identified excessive maternal weight gain during pregnancy as a known risk for infant weight gain (8). However, there remains minimal discussion across medical professions to collaboratively improve infant outcomes with prenatal interventions. Our focus has been limited to short-term outcomes, such as infant mortality, with little done to address long-term health risks persisting throughout childhood and into adult life. Policy and practice are entrenched in treating individuals rather than families, creating barriers in communication between parental and child providers that may limit the sharing of patient information and therapeutic strategies until it is too late to intervene.

It is also known that overweight in childhood is a significant risk factor for continued overweight into adulthood (9). Kuwahara et al. (7) illustrate the association between elevated BMI and growth in childhood and adolescence and higher serum uric acid levels into adulthood. The establishment of elevated inflammatory markers that are measurable in young adults herald the development of chronic disease as they move into adulthood. With increasing detail from cellular science at the ready, one can now point to numerous examples demonstrating mechanistic linkages between childhood processes and adult disease. It is essential that we use these connections to make clear for policy makers a need to invest in pediatric research that will lead to upstream health promotion and ultimately savings from the exorbitant costs of chronic adult disease. Although children are notoriously overlooked with respect to heath care costs due to their relatively small immediate impact on total health care expenditures, there is an argument that strengthened early identification and intervention may dramatically reduce the costs of poor health outcomes downstream.

Policy that understands the implications of lifespan science should target mechanisms that facilitate familial and intergenerational approaches to care. It is imperative that programs directed to parents, children, families, and all young adults (who are poised to become parents) be supported and expanded. The Maternal and Child Health Bureau has long been the epicenter for family-focused policies. The Title V Maternal and Child Health Block Grant program ensures funding for numerous essential programs to support women, children and adolescents, including prenatal and postnatal care for low-income women, and preventive health services focusing on reducing infant mortality and providing quality care for adolescents. The Title X Family Planning Program ensures access to family planning and preventive services. Likewise, a primary mission of Medicaid is to improve maternal and infant health outcomes. These programs are frequently at risk for underfunding or elimination with block grants and other mechanisms imposed to limit spending. Given the upcoming change in Federal administration, we as pediatric researchers are charged to advocate 
for our patients and present evidence of impact and successes in order to protect these essential programs and emphasize the benefits that an investment in child health will have on child and adult disease outcomes. This should be an utmost priority for all health professionals serving patients of any age.

Many more policies are currently in place and could be proposed to enhance intergenerational approaches to care: home visitation, family leave, early childhood education, child care and Head Start, and essential Federal nutrition programs: SNAP, WIC, the Child and Adult Care Food Program, national school breakfast and lunch, and the Summer Food Service program. With such a variety of opportunities to support families comes an equal necessity to articulate and further advance the proven value of these programs. The utilization of research such as that presented by Hammoud et al.(6) and Kuwahara et al.(7) is key to this objective. We have the capacity to spotlight the interdependence of children and families and the measurable sequence of childhood experience and health that lays down the biological and social patterns of their, and our, future.

The American Academy of Pediatrics recently released a Blueprint for Children (10), highlighting child health policy recommendations for the Federal government across agencies. With the incoming administration comes a critical opportunity to address fundamental needs of children. Informing policy with evidence of the interconnected health outcomes of mother and newborn and of the enduring effects of childhood growth and development on adult health and productivity defines a central role for lifespan science and research in shaping our future.

\section{REFERENCES}

1. Fox SE, Levitt P, Nelson CA 3rd. How the timing and quality of early experiences influence the development of brain architecture. Child Dev 2010;81:28-40.

2. Shonkoff JP, Garner AS. The Committee on Psychological Aspects of Child and Family Health, Committee on Early Childhood, Adoption, and Dependent Care, and Section on Developmental and Behavioral Pediatrics. The lifelong effects of early childhood adversity and toxic stress. Pediatrics 2012;129:232-46.

3. Bruyndonckx L, Hoymans VY, Lemmens K, Ramet J, Vrints CJ. Childhood obesity-related endothelial dysfunction: an update on pathophysiological mechanisms and diagnostic advancements. Pediatr Res 2016;79: 831-7.

4. Halfon N, Larson K, Lu M, Tullis E, Russ S. Lifecourse health development: past, present and future. Matern Child Health J 2014;18:344-65.

5. Wise PH. Child poverty and the promise of human capacity: childhood as a foundation for healthy aging. Acad Pediatr 2016;16(3 Suppl): S37-45.

6. Hammoud NM, de Valk HW, van Rossem L, Biesma DH, Wit JM, Visser GH. Growth and body mass index during the first 14 years of life in offspring from women with type 1 or type 2 diabetes mellitus. Pediatr Res 2016; e-pub ahead of print 09 November 2016.

7. Kuwahara E, Murakami Y, Okamura T, et al. Increased childhood body mass index is associated with young adult serum uric acid levels: a linkage study from Japan. Pediatr Res 2016; e-pub ahead of print 27 October 2016.

8. IOM (Institute of Medicine) and NRC (National Research Council). Weight Gain During Pregnancy: Reexamining the Guidelines. Washington, DC: The National Academies Press; 2009.

9. Biro FM, Wien M. Childhood obesity and adult morbidities. Am J Clin Nutr 2010;91:1499S-505S.

10. Blueprint for Children: How the next president can build a foundation for a healthy future. American Academy of Pediatrics. 2016. https://www.aap. org/en-us/Documents/BluePrintForChildren.pdf. 